\title{
A fast multipole accelerated BEM for 3-D elastic wave computation
}

\author{
Stéphanie Chaillat*** $^{*}$ Marc Bonnet* - Jean-François Semblat ${ }^{* *}$ \\ * Solid Mechanics Laboratory (UMR CNRS 7649), Department of Mechanics \\ École Polytechnique, F-91128 Palaiseau cedex, France \\ \{chaillat,bonnet\}@lms.polytechnique.fr \\ ** Université Paris-Est, Laboratoire Central des Ponts et Chaussées \\ 58 boulevard Lefebvre, 75015 Paris, France \\ semblat@lcpc.fr
}

\begin{abstract}
The solution of the elastodynamic equations using boundary element methods (BEMs) gives rise to fully-populated matrix equations. Earlier investigations on the Helmholtz and Maxwell equations have established that the Fast Multipole (FM) method reduces the complexity of a BEM solution to $\mathrm{N} \log _{2} \mathrm{~N}$ per GMRES iteration. The present article addresses the extension of the FM-BEM strategy to $3 D$ elastodynamics in the frequency domain. Efficiency and accuracy are demonstrated on numerical examples involving up to $N=O\left(10^{6}\right)$ boundary nodal unknowns.

RÉSUMÉ. La résolution des équations de l'élastodynamique par la méthode des éléments de frontière (BEM) conduit à un système linéaire plein. Faisant suite à des travaux sur les équations de Helmholtz et Maxwell ayant établi la capacité de la méthode multipôle rapide (FM) à réduire la complexité de la BEM à $N \log _{2} N$ par itération d'un solveur de type GMRES, cet article présente la transposition de l'approche FM-BEM à l'élastodynamique $3 D$ dans le domaine fréquentiel. La précision et l'efficacité de la méthode sont illustrées sur des exemples numériques mobilisant jusqu'à $N=O\left(10^{6}\right)$ inconnues nodales de frontière.

KEYWORDS: boundary element method; fast multipole method; 3D elastodynamics.

MOTS-CLÉS : méthode des éléments de frontière; méthode multipôle rapide; élastodynamique $3 D$.
\end{abstract}

$1^{\text {re }}$ soumission à $R E M N$, le 13 th October 2008 


\section{Introduction}

The boundary element method (BEM), pioneered in the sixties (Cruse, 1969; Rizzo, 1967), is a mesh reduction method, subject to restrictive constitutive assumptions but yielding highly accurate solutions. It is in particular well suited to deal with unbounded-domain idealizations commonly used in seismology (Dangla et al., 2005; Guzina et al., 2001) for example. In contrast with domain discretization methods, artificial boundary conditions (Givoli, 1992) are not needed for dealing with the radiation conditions, and grid dispersion cumulative effects are absent (Ihlenburg et al., 1995; Semblat et al., 2000).

However, in traditional boundary element (BE) implementations, the dimensional advantage with respect to domain discretization methods is offset by the fullypopulated nature of the BEM coefficient matrix, with set-up and solution times rapidly increasing with the problem size $N$. It is thus essential to develop alternative, faster strategies that allow to still exploit the known advantages of BEMs when large $N$ prohibit the use of traditional implementations.

In other areas such as computational electromagnetism or acoustics, considerable improvements in the computing speed and memory efficiency of BEM algorithms have been achieved on the basis of the Fast Multipole Method (FMM) (see the review article by Nishimura (2002)), with solution times typically of order $O\left(N \log _{2} N\right)$ per iteration for frequency-domain wave propagation problems (instead of $O\left(N^{2}\right)$ per iteration with traditional forms of the BEM).

This article is concerned with the formulation and implementation of a multilevel FM-BEM for 3-D elastodynamics in the frequency domain. Only a few references address this particular area of application. Two- and three-dimensional FM-BEMs for frequency-domain elastodynamics are proposed in (Fujiwara, 1998) and (Fujiwara, 2000; Yoshida, 2001), respectively, while time-domain problems are addressed in (Takahashi et al., 2003). The present work improves on the methodology of (Fujiwara, 2000) by incorporating recent advances of FMM implementations for Maxwell equations (Darve, 2000a) for achieving optimal computational efficiency. Both the single-level and multi-level forms of the FM-BEM are considered, with emphasis on the latter.

\section{Boundary integral method}

\subsection{Boundary integral representation}

Let $\Omega \subset \mathbb{R}^{3}$ denote the region of space occupied by a three-dimensional elastic solid with isotropic constitutive properties defined by $\mu$ (shear modulus), $\nu$ (Poisson's ratio) and $\rho$ (mass density). Time-harmonic motions, with circular frequency $\omega$, induced by a prescribed traction distribution $t^{D}$ on the boundary $\partial \Omega$ and in the absence of body forces, are considered for definiteness in this article. The accommodation of other boundary conditions requires only straight forward modifications to the treat- 
ment proposed therein. The displacement $\boldsymbol{u}$ is given at an interior point $\boldsymbol{x} \in \Omega$ by the following well-known representation formula (Bonnet, 1999):

$$
u_{k}(\boldsymbol{x})=-\int_{\partial \Omega} u_{i}(\boldsymbol{y}) T_{i}^{k}(\boldsymbol{x}, \boldsymbol{y} ; \omega) d S_{y}+\int_{\partial \Omega} t_{i}^{D}(\boldsymbol{y}) U_{i}^{k}(\boldsymbol{x}, \boldsymbol{y} ; \omega) d S_{y}(\boldsymbol{x} \in \Omega),
$$

where $U_{i}^{k}(\boldsymbol{x}, \boldsymbol{y} ; \omega)$ and $T_{i}^{k}(\boldsymbol{x}, \boldsymbol{y} ; \omega)$ denote the $i$-th components of the elastodynamic fundamental solution, i.e. of the displacement and traction, respectively, generated at $\boldsymbol{y} \in \mathbb{R}^{3}$ by a unit point force applied at $\boldsymbol{x} \in \mathbb{R}^{3}$ along the direction $k$, given by (Eringen et al., 1975):

$$
\begin{aligned}
U_{i}^{k}(\boldsymbol{x}, \boldsymbol{y} ; \omega) & =\frac{1}{k_{\mathrm{S}}^{2} \mu}\left(\left(\delta_{q s} \delta_{i k}-\delta_{q k} \delta_{i s}\right) \frac{\partial}{\partial x_{q}} \frac{\partial}{\partial y_{s}} G\left(|\boldsymbol{y}-\boldsymbol{x}| ; k_{\mathrm{S}}\right)\right. \\
& \left.+\frac{\partial}{\partial x_{i}} \frac{\partial}{\partial y_{k}} G\left(|\boldsymbol{y}-\boldsymbol{x}| ; k_{\mathrm{P}}\right)\right), \\
T_{i}^{k}(\boldsymbol{x}, \boldsymbol{y} ; \omega) & =C_{i j h \ell} \frac{\partial}{\partial y_{\ell}} U_{h}^{k}(\boldsymbol{x}, \boldsymbol{y} ; \omega) n_{j}(\boldsymbol{y}),
\end{aligned}
$$

where $k_{\mathrm{S}}$ and $k_{\mathrm{P}}$ are the respective wavenumbers of $\mathrm{S}$ and $\mathrm{P}$ elastic waves, so that

$$
k_{\mathrm{S}}^{2}=\frac{\rho \omega^{2}}{\mu}, \quad k_{\mathrm{P}}=\gamma k_{\mathrm{S}}, \quad \gamma^{2}=\frac{1-2 \nu}{2(1-\nu)},
$$

$G(\cdot ; k)$ is the free-space Green's function for the Helmholtz equation with wavenumber $k$, given by

$$
G(r ; k)=\frac{\exp (\mathrm{i} k r)}{4 \pi r},
$$

$\boldsymbol{n}(\boldsymbol{y})$ is the unit normal to $\partial \Omega$ directed outwards of $\Omega$, and $C_{i j h \ell}$ are the components of the fourth-order elasticity tensor, i.e.:

$$
C_{i j h \ell}=\mu\left[\frac{2 \nu}{1-2 \nu} \delta_{i j} \delta_{h \ell}+\delta_{i h} \delta_{j \ell}+\delta_{j h} \delta_{i \ell}\right] .
$$

\subsection{Boundary integral equation}

When $\boldsymbol{x} \in \partial \Omega$, a singularity occurs in $\boldsymbol{y}=\boldsymbol{x}$. With the help of a well-documented limiting process (Brebbia, 1984), the integral representation [1] yields the integral equation:

$$
(\mathcal{K} u)(\boldsymbol{x})=f(\boldsymbol{x}) \quad(\boldsymbol{x} \in \partial \Omega),
$$

with the linear integral operator $\mathcal{K}$ and the right-hand side $f$ defined by

$$
\begin{aligned}
(\mathcal{K} u)(\boldsymbol{x}) & =c_{i k}(\boldsymbol{x}) u_{i}(\boldsymbol{x})+(\text { P.V. }) \int_{\partial \Omega} u_{i}(\boldsymbol{y}) T_{i}^{k}(\boldsymbol{x}, \boldsymbol{y} ; \omega) d S_{y} \\
f(\boldsymbol{x}) & =\int_{\partial \Omega} t_{i}^{D}(\boldsymbol{y}) U_{i}^{k}(\boldsymbol{x}, \boldsymbol{y} ; \omega) d S_{y}
\end{aligned}
$$


where (P.V.) indicates a Cauchy principal value (CPV) singular integral and the freeterm $c_{i k}(\boldsymbol{x})$ is equal to $0.5 \delta_{i k}$ in the usual case where $\partial \Omega$ is smooth at $\boldsymbol{x}$. The integral operator [7] may be recast into alternative, equivalent regularized forms which are free of CPV integrals (Bonnet, 1999). Equations [1] and [6] are applicable to either interior or exterior elastodynamic problems.

\section{Fast Multipole Method: principle}

\subsection{Multipole expansions of the elastodynamic fundamental solutions}

The FMM is based on a reformulation of the fundamental solutions in terms of products of functions of $\boldsymbol{x}$ and of $\boldsymbol{y}$. This allows to re-use integrations with respect to $\boldsymbol{y}$ when the collocation point $\boldsymbol{x}$ is changed, thereby lowering the $O\left(N^{2}\right)$ complexity per iteration entailed by standard BEMs. The elastodynamic fundamental solutions [2a,b] are linear combinations of derivatives of the Green's function [4] for the Helmholtz equation. On recasting the position vector $\boldsymbol{r}=\boldsymbol{y}-\boldsymbol{x}$ in the form $\boldsymbol{r}=\boldsymbol{r}_{0}+\left(\boldsymbol{y}-\boldsymbol{y}_{0}\right)-$ $\left(\boldsymbol{x}-\boldsymbol{x}_{0}\right)$, where $\boldsymbol{x}_{0}$ and $\boldsymbol{y}_{0}$ are two poles and $\boldsymbol{r}_{0}=\boldsymbol{y}_{0}-\boldsymbol{x}_{0}$, the Helmholtz Green's function is shown (Epton et al., 1995; Darve, 2000b) to admit the decomposition

$$
G(|\boldsymbol{r}| ; k)=\lim _{L \rightarrow+\infty} \int_{\hat{\boldsymbol{s}} \in S} e^{-\mathrm{i} k \hat{\boldsymbol{s}} \cdot\left(\boldsymbol{y}-\boldsymbol{y}_{0}\right)} \mathcal{G}_{L}\left(\hat{\boldsymbol{s}} ; \boldsymbol{r}_{0} ; k\right) e^{\mathrm{i} k \hat{\boldsymbol{s}} \cdot\left(\boldsymbol{x}-\boldsymbol{x}_{0}\right)} \mathrm{d} \hat{\boldsymbol{s}}
$$

where $S$ is the unit sphere of $\mathbb{R}^{3}$ and the transfer function $\mathcal{G}_{L}\left(\hat{\boldsymbol{s}} ; \boldsymbol{r}_{0} ; k\right)$ is defined in terms of the Legendre polynomials $P_{p}$ and the spherical Hankel functions of the first kind $h_{p}^{(1)}$ by:

$$
\mathcal{G}_{L}\left(\hat{\boldsymbol{s}} ; \boldsymbol{r}_{0} ; k\right)=\frac{\mathrm{i} k}{16 \pi^{2}} \sum_{0 \leq p \leq L}(2 p+1) \mathrm{i}^{p} h_{p}^{(1)}\left(k\left|\boldsymbol{r}_{0}\right|\right) P_{p}\left(\cos \left(\hat{\boldsymbol{s}}, \boldsymbol{r}_{0}\right)\right) .
$$

The decomposition [9]-[10] is seen to achieve the desired separation of variables $\boldsymbol{x}$ and $\boldsymbol{y}$. A similar multipole decomposition of the elastodynamic fundamental solutions is easily obtained:

$$
\begin{aligned}
U_{i}^{k}(\boldsymbol{x}, \boldsymbol{y} ; \omega)= & \lim _{L \rightarrow+\infty} \int_{\hat{\boldsymbol{s}} \in S} e^{-\mathrm{i} k_{\mathrm{P}} \hat{\boldsymbol{s}} \cdot\left(\boldsymbol{y}-\boldsymbol{y}_{0}\right)} \mathcal{U}_{i, L}^{k, \mathrm{P}}\left(\hat{\boldsymbol{s}} ; \boldsymbol{r}_{0}\right) e^{\mathrm{i} k_{\mathrm{P}} \hat{\boldsymbol{s}} \cdot\left(\boldsymbol{x}-\boldsymbol{x}_{0}\right)} \mathrm{d} \hat{\boldsymbol{s}} \\
& +\lim _{L \rightarrow+\infty} \int_{\hat{\boldsymbol{s}} \in S} e^{-\mathrm{i} k_{\mathrm{S}} \hat{\boldsymbol{s}} \cdot\left(\boldsymbol{y}-\boldsymbol{y}_{0}\right)} \mathcal{U}_{i, L}^{k, \mathrm{~S}}\left(\hat{\boldsymbol{s}} ; \boldsymbol{r}_{0}\right) e^{\mathrm{i} k_{\mathrm{S}} \hat{\boldsymbol{s}} \cdot\left(\boldsymbol{x}-\boldsymbol{x}_{0}\right)} \mathrm{d} \hat{\boldsymbol{s}}, \\
T_{i}^{k}(\boldsymbol{x}, \boldsymbol{y} ; \omega)= & \lim _{L \rightarrow+\infty} \int_{\hat{\boldsymbol{s}} \in S} e^{-\mathrm{i} k_{\mathrm{P}} \hat{\boldsymbol{s}} \cdot\left(\boldsymbol{y}-\boldsymbol{y}_{0}\right)} \mathcal{T}_{i, L}^{k, \mathrm{P}}\left(\hat{\boldsymbol{s}} ; \boldsymbol{r}_{0}\right) e^{\mathrm{i} k_{\mathrm{P}} \hat{\boldsymbol{s}} \cdot\left(\boldsymbol{x}-\boldsymbol{x}_{0}\right)} \mathrm{d} \hat{\boldsymbol{s}} \\
& +\lim _{L \rightarrow+\infty} \int_{\hat{\boldsymbol{s}} \in S} e^{-\mathrm{i} k_{\mathrm{S}} \hat{\boldsymbol{s}} \cdot\left(\boldsymbol{y}-\boldsymbol{y}_{0}\right)} \mathcal{T}_{i, L}^{k, \mathrm{~S}}\left(\hat{\boldsymbol{s}} ; \boldsymbol{r}_{0}\right) e^{\mathrm{i} k_{\mathrm{S}} \hat{\boldsymbol{s}} \cdot\left(\boldsymbol{x}-\boldsymbol{x}_{0}\right)} \mathrm{d} \hat{\boldsymbol{s}},
\end{aligned}
$$


with the elastodynamic transfer functions given in terms of the acoustic transfer function $\mathcal{G}_{L}$ by

$$
\begin{gathered}
\mathcal{U}_{i, L}^{k, \mathrm{P}}\left(\hat{\boldsymbol{s}} ; \boldsymbol{r}_{0}\right)=\frac{\gamma^{2}}{\mu} \hat{s}_{i} \hat{s}_{k} \mathcal{G}_{L}\left(\hat{\boldsymbol{s}} ; \boldsymbol{r}_{0} ; k_{\mathrm{P}}\right), \\
\mathcal{T}_{i, L}^{k, \mathrm{P}}\left(\hat{\boldsymbol{s}} ; \boldsymbol{r}_{0}\right)=\frac{-\mathrm{i} k_{\mathrm{S}} \gamma^{3}}{\mu} C_{i j h \ell} \hat{s}_{\ell} \hat{s}_{h} \hat{s}_{k} \mathcal{G}_{L}\left(\hat{\boldsymbol{s}} ; \boldsymbol{r}_{0} ; k_{\mathrm{P}}\right) n_{j}(\boldsymbol{y}), \\
\mathcal{U}_{i, L}^{k, \mathrm{~S}}\left(\hat{\boldsymbol{s}} ; \boldsymbol{r}_{0}\right)=\frac{1}{\mu}\left(\delta_{i k}-\hat{s}_{k} \hat{s}_{i}\right) \mathcal{G}_{L}\left(\hat{\boldsymbol{s}} ; \boldsymbol{r}_{0} ; k_{\mathrm{S}}\right), \\
\mathcal{T}_{i, L}^{k, \mathrm{~S}}\left(\hat{\boldsymbol{s}} ; \boldsymbol{r}_{0}\right)=\frac{-\mathrm{i} k_{\mathrm{S}}}{\mu}\left(\delta_{h k}-\hat{s}_{k} \hat{s}_{h}\right) C_{i j h \ell} \hat{s}_{\ell} \mathcal{G}_{L}\left(\hat{\boldsymbol{s}} ; \boldsymbol{r}_{0} ; k_{\mathrm{S}}\right) n_{j}(\boldsymbol{y}) .
\end{gathered}
$$

In practice, the limiting process $L \rightarrow+\infty$ in [9] or [11], [12] cannot be performed exactly and is replaced with an evaluation for a suitably chosen finite value of $L$. A key error analysis result (Darve, 2000b) states that there exist four constants $C_{1}, C_{2}, C_{3}, C_{4}$ such that

$$
\begin{aligned}
L= & C_{1}+C_{2} k\left|\boldsymbol{r}-\boldsymbol{r}_{0}\right|+C_{3} \ln \left(k\left|\boldsymbol{r}-\boldsymbol{r}_{0}\right|\right)+C_{4} \ln \epsilon^{-1} \\
& \Longrightarrow\left|\frac{\exp (\mathrm{i} k|\boldsymbol{r}|)}{4 \pi|\boldsymbol{r}|}-\int_{\hat{\boldsymbol{s}} \in S} e^{-\mathrm{i} k \hat{\boldsymbol{s}} \cdot\left(\boldsymbol{y}-\boldsymbol{y}_{0}\right)} \mathcal{G}_{L}\left(\hat{\boldsymbol{s}} ; \boldsymbol{r}_{0} ; k\right) e^{\mathrm{i} k \hat{\boldsymbol{s}} \cdot\left(\boldsymbol{x}-\boldsymbol{x}_{0}\right)} \mathrm{d} \hat{\boldsymbol{s}}\right|<\epsilon
\end{aligned}
$$

for any chosen error level $\epsilon<1$, whenever

$$
\left|\boldsymbol{r}-\boldsymbol{r}_{0}\right| /\left|\boldsymbol{r}_{0}\right| \leq 2 / \sqrt{5} \text {. }
$$

The result [15], [16] implies that expansions [11], [12] must be used for wellseparated sets of collocation and integration points clustered around poles $\boldsymbol{x}_{0}$ and $\boldsymbol{y}_{0}$.

\subsection{Single-level fast multipole formulation}

In the single-level FMM, a 3D cubic grid of linear spacing $d$ embedding the boundary $\partial \Omega$ is introduced. The centers of the cubic cells thus defined are taken as poles $\boldsymbol{x}_{0}$ or $\boldsymbol{y}_{0}$ in decompositions [11], [12]. Two cells are deemed adjacent (letting $\mathcal{A}(\mathcal{C})$ denote the set of cells which are adjacent to a given cubic cell $\mathcal{C}$ ), if they have at least one common point, e.g. a vertex. Whenever $\boldsymbol{x}$ and $\boldsymbol{y}$ belong to cells $\mathcal{C}_{x}$ and $\mathcal{C}_{y}$ that are not adjacent, condition [16] is automatically fulfilled and expansions [11], [12] can be safely used. Conversely, when $\boldsymbol{x}$ and $\boldsymbol{y}$ lie in adjacent cells, condition [16] is not assured and the classical expressions [2a,b] of the fundamental solutions are used instead. These considerations lead to reformulate expressions [7] and [8], for any collocation point $\boldsymbol{x}$ lying in a given cell $\mathcal{C}_{x}$, as

$$
\begin{aligned}
(\mathcal{K} u)(\boldsymbol{x}) & =(\mathcal{K} u)^{\text {near }}(\boldsymbol{x})+(\mathcal{K} u)^{\mathrm{FM}}(\boldsymbol{x}), & \\
f(\boldsymbol{x}) & =f^{\text {near }}(\boldsymbol{x})+f^{\mathrm{FM}}(\boldsymbol{x}) & \left(\boldsymbol{x} \in \partial \Omega \cap \mathcal{C}_{x}\right),
\end{aligned}
$$


where the "near" parts are defined for each collocation point $\boldsymbol{x}$ as the net contributions from the portion of boundary situated in cells adjacent to that containing $\boldsymbol{x}$. The "FM" parts then collect all contributions from cells that are not adjacent to $\mathcal{C}_{x}$.

The "near" contributions are evaluated by means of standard BE techniques. The treatment of the "FM" contributions exploits expansions [11], [12] truncated at a finite $L$ and in a manner suggested by their multiplicative form, i.e. (i) evaluate integrals over each cell $\mathcal{C}_{y}$ and associate obtained values to the cell center $\boldsymbol{y}_{0}$, (ii) apply transfer functions to obtain quantities associated to the center $\boldsymbol{x}_{0}$ of cell $\mathcal{C}_{x}$, and (iii) evaluate contribution at each collocation point $\boldsymbol{x} \in \mathcal{C}_{x}$. Accordingly, multipole moments, defined by

$$
\begin{aligned}
\mathcal{R}_{k}^{\mathrm{S}, u}\left(\hat{\boldsymbol{s}} ; \mathcal{C}_{y}\right) & =-\mathrm{i} k_{\mathrm{S}}\left[\delta_{i k} \hat{s}_{j}+\delta_{j k} \hat{s}_{i}-2 \hat{s}_{i} \hat{s}_{j} \hat{s}_{k}\right] \\
& \times \int_{\partial \Omega \cap \mathcal{C}_{y}} u_{i}(\boldsymbol{y}) n_{j}(\boldsymbol{y}) e^{-\mathrm{i} k_{\mathrm{S}} \hat{\boldsymbol{s}} \cdot\left(\boldsymbol{y}-\boldsymbol{y}_{0}\right)} d S_{\tilde{y}} \\
\mathcal{R}^{\mathrm{P}, u}\left(\hat{\boldsymbol{s}} ; \mathcal{C}_{y}\right) & =-\mathrm{i} k_{\mathrm{S}} \gamma^{3}\left[\frac{2 \nu}{1-2 \nu} \delta_{i j}+2 \hat{s}_{i} \hat{s}_{j}\right] \\
& \times \int_{\partial \Omega \cap \mathcal{C}_{y}} u_{i}(\boldsymbol{y}) n_{j}(\boldsymbol{y}) e^{-\mathrm{i} k_{\mathrm{P}} \hat{\boldsymbol{s}} \cdot\left(\boldsymbol{y}-\boldsymbol{y}_{0}\right)} d S_{\tilde{y}} \\
\mathcal{R}_{k}^{\mathrm{S}, t}\left(\hat{\boldsymbol{s}} ; \mathcal{C}_{y}\right)= & \frac{1}{\mu}\left[\delta_{k a}-\hat{s}_{k} \hat{s}_{a}\right] \int_{\partial \Omega \cap \mathcal{C}_{y}} t_{a}(\boldsymbol{y}) e^{-\mathrm{i} k_{\mathrm{S}} \hat{\boldsymbol{s}} \cdot\left(\boldsymbol{y}-\boldsymbol{y}_{0}\right)} d S_{\tilde{y}} \\
\mathcal{R}^{\mathrm{P}, t}\left(\hat{\boldsymbol{s}} ; \mathcal{C}_{y}\right)= & \frac{\gamma^{2}}{\mu} \int_{\partial \Omega \cap \mathcal{C}_{y}} \hat{s}_{a} t_{a}(\boldsymbol{y}) e^{-\mathrm{i} k_{\mathrm{P}} \hat{\boldsymbol{s}} \cdot\left(\boldsymbol{y}-\boldsymbol{y}_{0}\right)} d S_{\tilde{y}}
\end{aligned}
$$

are computed for each cell $\mathcal{C}_{y}$ (step (i)). Then, local expansions for the cell $\mathcal{C}_{x}$ are evaluated by applying the transfer functions to the multipole moments according to

$$
\begin{aligned}
\mathcal{L}_{k}^{\mathrm{S}, u}\left(\hat{\boldsymbol{s}} ; \mathcal{C}_{x}\right) & =\sum_{\mathcal{C}_{y} \notin \mathcal{A}\left(\mathcal{C}_{x}\right)} \mathcal{G}_{L}\left(\hat{\boldsymbol{s}} ; \boldsymbol{r}_{0} ; k_{\mathrm{S}}\right) \mathcal{R}_{k}^{\mathrm{S}, u}\left(\hat{\boldsymbol{s}} ; \mathcal{C}_{y}\right), \\
\mathcal{L}^{\mathrm{P}, u}\left(\hat{\boldsymbol{s}} ; \mathcal{C}_{x}\right) & =\sum_{\mathcal{C}_{y} \notin \mathcal{A}\left(\mathcal{C}_{x}\right)} \mathcal{G}_{L}\left(\hat{\boldsymbol{s}} ; \boldsymbol{r}_{0} ; k_{\mathrm{P}}\right) \mathcal{R}^{\mathrm{P}, u}\left(\hat{\boldsymbol{s}} ; \mathcal{C}_{y}\right) \\
\mathcal{L}_{k}^{\mathrm{S}, t}\left(\hat{\boldsymbol{s}} ; \mathcal{C}_{x}\right) & =\sum_{\mathcal{C}_{y} \notin \mathcal{A}\left(\mathcal{C}_{x}\right)} \mathcal{G}_{L}\left(\hat{\boldsymbol{s}} ; \boldsymbol{r}_{0} ; k_{\mathrm{S}}\right) \mathcal{R}_{k}^{\mathrm{S}, t}\left(\hat{\boldsymbol{s}} ; \mathcal{C}_{y}\right), \\
\mathcal{L}^{\mathrm{P}, t}\left(\hat{\boldsymbol{s}} ; \mathcal{C}_{x}\right) & =\sum_{\mathcal{C}_{y} \notin \mathcal{A}\left(\mathcal{C}_{x}\right)} \mathcal{G}_{L}\left(\hat{\boldsymbol{s}} ; \boldsymbol{r}_{0} ; k_{\mathrm{P}}\right) \mathcal{R}^{\mathrm{P}, t}\left(\hat{\boldsymbol{s}} ; \mathcal{C}_{y}\right)
\end{aligned}
$$

where $\boldsymbol{r}_{0}=\boldsymbol{y}_{0}-\boldsymbol{x}_{0}$ joins the centers of cells $\mathcal{C}_{x}$ and $\mathcal{C}_{y}$ (step (ii)). Upon multiplying [20a,b], [21a,b] by the local factors $\exp \left[\mathrm{i} k_{\alpha} \hat{\boldsymbol{s}} .\left(\boldsymbol{x}-\boldsymbol{x}_{0}\right)\right]$ (step (iii)) and replacing the integration over the unit sphere in [11], [12] by a numerical quadrature rule based 
on a set of $Q$ quadrature points $\hat{\boldsymbol{s}}_{q} \in S$ and weights $w_{q}$, the "FM" contributions finally take the form

$$
\begin{aligned}
&(\mathcal{K} u)_{k}^{\mathrm{FM}}(\boldsymbol{x}) \approx \sum_{q=1}^{Q} w_{q}\left[e^{\mathrm{i} k_{\mathrm{S}} \hat{\boldsymbol{s}}_{q} \cdot\left(\boldsymbol{x}-\boldsymbol{x}_{0}\right)} \mathcal{L}_{k}^{\mathrm{S}, u}\left(\hat{\boldsymbol{s}}_{q} ; \mathcal{C}_{x}\right)\right. \\
&\left.+e^{\mathrm{i} k_{\mathrm{P}} \hat{\boldsymbol{s}}_{q} \cdot\left(\boldsymbol{x}-\boldsymbol{x}_{0}\right)}\left(\hat{\boldsymbol{s}}_{q}\right)_{k} \mathcal{L}^{\mathrm{P}, u}\left(\hat{\boldsymbol{s}}_{q} ; \mathcal{C}_{x}\right)\right] \\
& f_{k}^{\mathrm{FM}}(\boldsymbol{x}) \approx \sum_{q=1}^{Q} w_{q}\left[e^{\mathrm{i} k_{\mathrm{S}} \hat{\boldsymbol{s}}_{q} \cdot\left(\boldsymbol{x}-\boldsymbol{x}_{0}\right)} \mathcal{L}_{k}^{\mathrm{S}, t}\left(\hat{\boldsymbol{s}}_{q} ; \mathcal{C}_{x}\right)\right. \\
&\left.+e^{\mathrm{i} k_{\mathrm{P}} \hat{\boldsymbol{s}}_{q} \cdot\left(\boldsymbol{x}-\boldsymbol{x}_{0}\right)}\left(\hat{\boldsymbol{s}}_{q}\right)_{k} \mathcal{L}^{\mathrm{P}, t}\left(\hat{\boldsymbol{s}}_{q} ; \mathcal{C}_{x}\right)\right]
\end{aligned}
$$

Expression [22] defines the "FM" contribution to the matrix-vector product $[K]\{u\}$, and hence is evaluated once per GMRES iteration, while [23] provides the "FM" contribution to the right-hand side $\{f\}$ and is computed once, prior to calling the GMRES solver.

The single-level elastodynamic FMM is more efficient than the classical BEM, with a complexity of $O\left(N^{3 / 2}\right)$ per GMRES iteration. Further acceleration is achievable by adopting a multi-level approach, as described next for the present context of 3-D elastodynamics.

\subsection{Multi-level fast multipole formulation}

To have maximal efficiency, FM-BEM algorithms must confine non-FM calculations to the smallest possible portion of the boundary while clustering whenever possible the computation of influence terms into the largest possible non-adjacent groups. This is achieved by the multi-level FMM (Darve, 2000a; Nishimura, 2002; Sylvand, 2002), which is based on using large cells and hierarchically subdividing each cell into $2 \times 2 \times 2=8$ children cubic cells. This cell-subdivision approach is systematized by means of an oct-tree structure of cells. The level $\ell=0$, composed of only one cubic cell containing the whole surface $\partial \Omega$, is the tree root. The level- 0 cell is divided into $2 \times 2 \times 2=8$ children cubic cells, which constitute the level $\ell=1$. All level- 1 cells being adjacent, the FMM cannot be applied to them. The level $\ell=2$ is then defined by dividing each level- 1 cell into 8 children cells, and so contains 64 cells. The subdivision process is further repeated until the finest level $\ell=\bar{\ell}$, implicitly defined by a preset subdivision-stopping criterion, is reached. Level- $\bar{\ell}$ cells are usually termed leaf cells.

The multi-level approach basically consists in first applying the FMM to all influence computations between disjoint level-2 cells (so as to use the largest clusters whenever possible), and then recursively tracing the tree downwards, applying the FMM to all interaction between disjoint level- $\ell$ cells that are children of adjacent level- $(\ell-1)$ cells. Finally, interactions between adjacent leaf cells are treated using traditional (i.e. non FM-based) BE techniques. This approach thus minimizes the overall proportion of influence computations requiring the traditional treatment. 
Previous studies for the Maxwell equations (Darve, 2000a) have established a crucial practical consideration: for a given desired accuracy, both the truncation parameter $L$ in the transfer function [10] and the number of quadrature points $\tilde{\boldsymbol{s}}_{q}$ depend on the subdivision level, and in fact increase with $k_{\alpha} d$ (the cell-size-to-wavelength ratio). The present implementation incorporates these findings, whereas that of Fujiwara (2000) does not. Accounting for these effects, the theoretical complexity of the multi-level FMM is $O\left(N \log _{2} N\right)$ per iteration both for CPU time and memory (i.e. somewhat higher than the $O(N)$ complexity for static FM-BEM, where the truncation parameter in the FMM expansion is not level-dependent).

\section{Fast Multipole Method: accuracy and computational efficiency}

Both the single-level and multi-level elastodynamic FMM have been implemented, for three-noded triangular boundary elements. All examples have been run on the same single-processor PC (RAM: 3Go, CPU frequency: $3.40 \mathrm{GHz}$ ). Except where indicated otherwise, the multi-level FMM is used.

\subsection{Spherical cavity under internal pressure}

A spherical cavity of radius $R$ embedded in an elastic isotropic infinite medium (with $\nu=0.25$ ) is subjected to an internal time-harmonic uniform pressure $P$. This problem has a simple, spherically-symmetric, exact solution (Eringen et al., 1975) against which numerically-computed solutions are compared using the root mean square solution error. The results of such comparisons, performed for several normalized frequencies $\eta_{\mathrm{P}}=k_{\mathrm{P}} R$ (i.e. $\eta_{\mathrm{P}} / \pi$ is the number of $\mathrm{P}$ wavelengths spanned by the sphere diameter) and various distances from the cavity wall $(R<r \leq 3 R)$ are presented in Table 1 . The present FM-BEM is seen to be very accurate, even in the nearly-
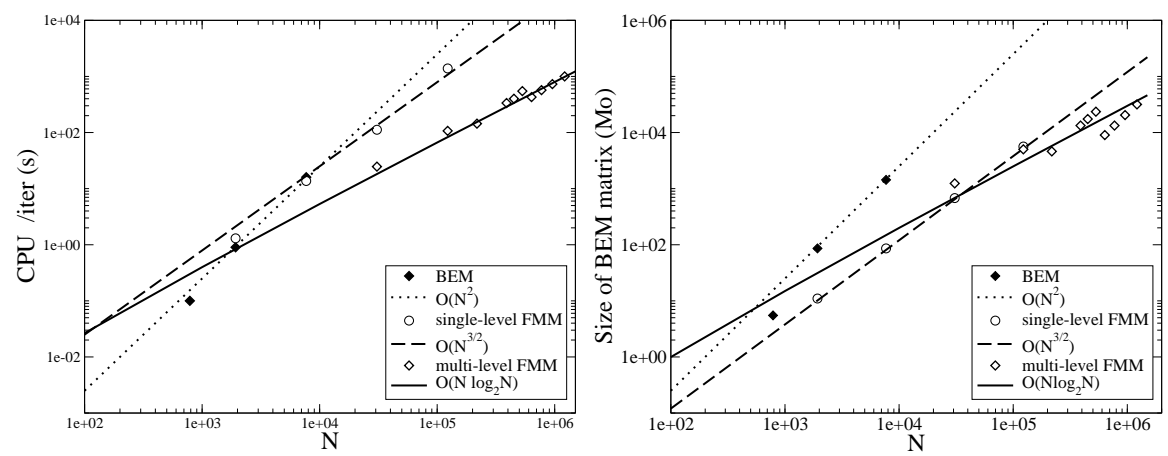

Figure 1. Complexity of the standard BEM, single-level FMM and multi-level FMM (left: CPU time, right: memory) 


\begin{tabular}{|c|c|c|c|c|}
\hline$\eta_{\mathrm{P}}=k_{\mathrm{P}} R / \pi$ & 0.01 & 0.50 & 1.00 & 2.00 \\
\hline$\#$ nodes $/ \lambda_{\mathrm{S}}$ & 800 & 16 & 8 & 4 \\
\hline RMS error, $r=R$ (cavity wall) & 0.018 & 0.006 & 0.006 & 0.021 \\
\hline RMS error, $R<r \leq 3 R$ (domain) & 0.017 & 0.006 & 0.008 & 0.031 \\
\hline
\end{tabular}

Table 1. Pressurized spherical cavity: RMS solution error on the cavity and in the domain

static case $\left(\eta_{\mathrm{P}}=0.01\right)$ for which the accuracy of FMM expansions of the form [9] is known to deteriorate (Darve, 2000a), whereas the standard BEM does not (Dangla et al., 2005). Moreover, the accuracy is seen to deteriorate, as expected, for mesh densities below about eight nodes per $\mathrm{S}$ wabelength. In all subsequent results, the meshes are designed so as to feature at least 10 nodes per $\mathrm{S}$ wavelength. Finally, the theoretical complexities (i.e. the CPU time spent for each GMRES iteration as a function of $N$ ) are now compared against recorded CPU times, on the pressurized spherical cavity problem. The numerical experiments corroborate previously mentioned theoretical complexity estimates for standard BEM, single-level FMM and multi-level FMM, as seen in Figure 1 . This complexity study involves problem sizes of up to $N \approx 1.210^{6}$, while the examples of Fujiwara (2000) used $N \leq 2.510^{4}$.

\subsection{Diffraction of an incident $P$ plane wave by a semi-spherical canyon}

This example is concerned with the diffraction by a semi-spherical canyon of a plane P-wave of unit amplitude travelling vertically in an elastic homogeneous irregular half-space (Figure 2), with again $\nu=0.25$. The semi-spherical surface of the canyon and the surrounding portion of free surface lying inside a disk of radius $D>R$ are discretized using boundary elements. Such a configuration is representative of a

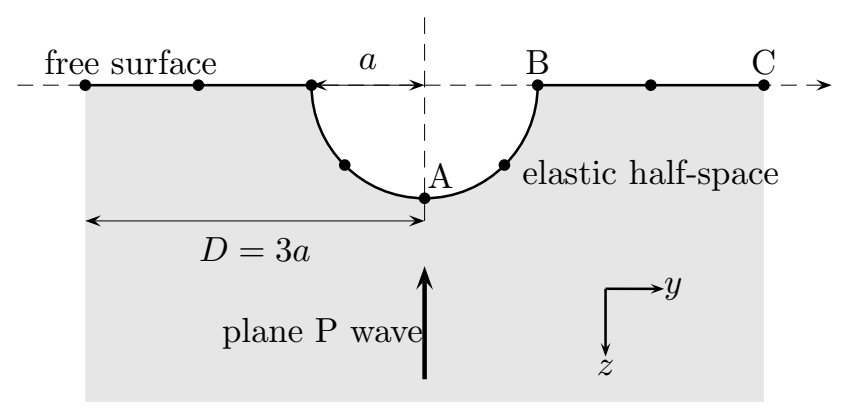

Figure 2. Diffraction of an incident P plane wave by a semi-spherical canyon: notation 


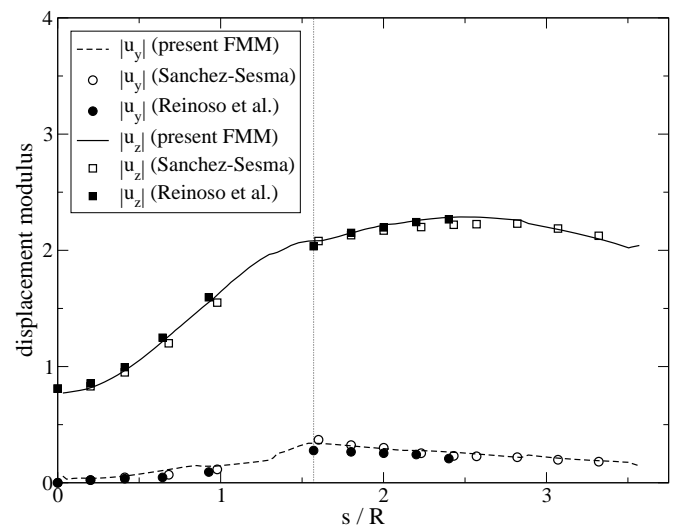

Figure 3. Diffraction of an incident $P$ plane wave by a semi-spherical canyon: horizontal and vertical computed displacement on line $A B C$ (with points $A, B, C$ defined on Fig. 2) plotted against arc-length abscissa s along $A B C$ (normalized frequency $\left.\eta_{P}=0.25\right)$

"topographic site effect" in seismology, and has been the subject of numerous studies, see (Dangla et al., 2005; Sánchez-Sesma, 1983) where diffraction of waves by surface heterogeneities is considered.

Here, results obtained by the present FM-BEM for the (low) normalized frequency $\eta_{\mathrm{P}}=0.25$, by means of a BE mesh featuring $N=23382$ DOFs, are compared to corresponding results from Sánchez-Sesma (1983) (based on a semi-analytical approach) and Reinoso et al. (1997) (obtained using a standard elastodynamic BEM). Figure 3 shows that the horizontal and vertical displacements on line $\mathrm{ABC}$ (with points $\mathrm{A}, \mathrm{B}$, $\mathrm{C}$ defined on Fig. 2) produced by the three approaches are in good agreement. Note that the corresponding results in Sánchez-Sesma (1983) and Reinoso et al. (1997) are plotted against the horizontal coordinate $y$, whereas the arc-length coordinate $s$ along ABC is used in Fig. 3. The same value $D=3 R$ of the truncation radius has been used for all three sets of results. The present computation required 7 GMRES iterations and $24 \mathrm{~s}$ of CPU time per iteration.

Moreover, the FM-BEM allows to deal with non-dimensional frequencies significantly higher than those considered in previous studies. Figure 4 shows the displacements along line $\mathrm{ABC}$ computed for a nondimensional frequency $\eta_{\mathrm{P}}=5$ using the present method. This time, the problem size $N=287946$ is well beyond the capabilities of standard BEM. This computation required 86 GMRES iterations (without preconditioning) and $5 \mathrm{mn}$ CPU time per iteration.

The displacement near the canyon edge (i.e. $y=R$ and $s=\pi R / 2$, see Fig. 2) has strong variations, as expected. 


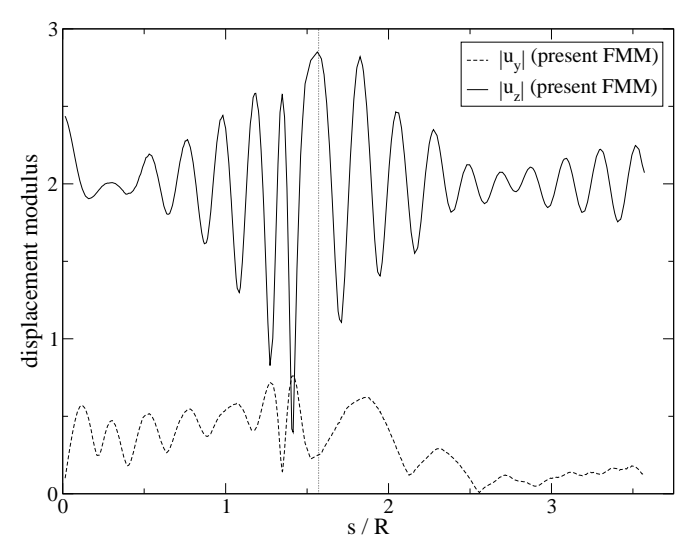

Figure 4. Diffraction of an incident $P$ plane wave by a semi-spherical canyon: horizontal and vertical computed displacement on line $A B C$ (with points $A, B, C$ defined on Fig. 2) plotted against arc-length abscissa s along $A B C$ (normalized frequency $\left.\eta_{P}=5\right)$

The size of the problems that can be solved is now limited by the number of iterations of the iterative solver. Reducing the iteration count requires a preconditioning strategy. This critical component of the development of efficient FM-BEM algorithms remains in the authors' view a largely open issue and is not addressed here.

\section{Conclusion}

In this article, the Fast Multipole Method has been succesfully extended to $3 D$ elastodynamics in the frequency domain. Combined with the BEM formulation, it permits to reduce the computational burden, in both CPU time and memory requirements, for the analysis of wave propagation (e. g. seismic), and allows to run BEM models of size $N=O\left(10^{6}\right)$ on an ordinary PC. Comparisons with analytical or previously published numerical results show the efficiency and accuracy of the present elastodynamic FM-BEM. Theoretical complexity estimates for both the single-level and multi-level formulations were derived and corroborated by numerical experiments.

Applications of the present FM-BEM to realistic cases in seismology are under way. Moreover, a natural extension of this work consists in formulating multipole expansions of other fundamental solutions, with the half-space elastodynamic fundamental solution being currently investigated.

\section{Acknowledgements}

This work is part of the project Quantitative Seismic Hazard Assessment (QSHA) funded by the French National Research Agency (ANR). 


\section{References}

Bonnet M., Boundary Integral Equation Method for Solids and Fluids, Wiley, 1999.

Brebbia C. A.and Telles J. C. F. W. L. C., Boundary element techniques, Springer, 1984.

Cruse T., " Numerical solutions in three-dimensional elastostatics", Int. J. Solids Struct., vol. 5, p. 1259-1274, 1969.

Dangla P., Semblat J. F., Xiao H., Delépine N., “ A simple and efficient regularization method for 3D BEM: application to frequency-domain elastodynamics", Bull. Seism. Soc. Am., vol. 95, p. 1916-1927, 2005.

Darve E., “The Fast Multipole Method : Numerical Implementation”, J. Comp. Phys., vol. 160, p. 195-240, 2000a.

Darve E., " The fast multipole method: I. Error analysis and asymptotic complexity”, SIAM J. Numer. Anal., vol. 38, p. 98-128, 2000b.

Epton M., Dembart B., “ Multipole translation theory for the three-dimensional Laplace and Helmholtz equations”, SIAM J. Sci. Comp., vol. 16, p. 865-897, 1995.

Eringen A., Suhubi E., Elastodynamics, vol. II-linear theory, Academic Press, 1975.

Fujiwara H., " The fast multipole method for the integral equations of seismic scattering problems”, Geophys. J. Int., vol. 133, p. 773-782, 1998.

Fujiwara H., " The fast multipole method for solving integral equations of three-dimensional topography and basin problems", Geophys. J. Int., vol. 140, p. 198-210, 2000.

Givoli D., Numerical Methods for Problems in infinite domains, Elsevier, 1992.

Guzina B. B., Pak R. Y. S., " On the Analysis of Wave Motions in a Multi-Layered Solid", Quart. J. Mech. Appl. Math., vol. 54, p. 13-37, 2001.

Ihlenburg F., Babuška I., “ Dispersion analysis and error estimation of Galerkin finite element methods for the Helmholtz equation”, Int. J. Numer. Meth. Engng., vol. 38, p. 3745-3774, 1995.

Nishimura N., " Fast multipole accelerated boundary integral equation methods", Appl. Mech. Rev., 2002.

Reinoso E., Wrobel L. C., Power H., “ Three-dimensional scattering of seismic waves from topographical structures”, Soil. Dyn. Earthquake Engng., vol. 16, p. 41-61, 1997.

Rizzo F., " An integral equation approach to boundary value problems of classical elastostatics", Quart. Appl. Math., vol. 25, p. 83-95, 1967.

Sánchez-Sesma F. J., “ Diffraction of elastic waves by 3D surface irregularities”, Bull. Seism. Soc. Am., vol. 73, p. 1621-1636, 1983.

Semblat J. F., Brioist J., “ Efficiency of higher order finite elements for the analysis of seismic wave propagation", J. Sound Vib., vol. 231, p. 460-467, 2000.

Sylvand G., La méthode multipôle rapide en éléctromagnétisme : performances, parallélisation, applications, PhD thesis, ENPC, 2002.

Takahashi T., Nishimura N., Kobayashi S., “ A fast BIEM for three-dimensional elastodynamics in time domain”, Engng. Anal. Bound. Elem., vol. 27, p. 491-506, 2003.

Yoshida K., Applications of fast multipole method to boundary integral equation method, $\mathrm{PhD}$ thesis, University of Kyoto, 2001. 